\title{
Medial Abrasion Syndrome: A Neglected Cause of Persistent Pain After Knee Arthroplasty
}

\section{Shaw-Ruey Lyu ( $D$ srlyu@mac.com )}

Dalin Tzu Chi General Hospital: Dalin Tzu Chi Hospital https://orcid.org/0000-0003-3797-4848

\section{Chia-Chen Hsu}

Dalin Tzu-Chi Hospital

\section{Jung-Pin Hung}

Dalin Tzu Chi Hospital

\section{Research article}

Keywords: medial abrasion syndrome, arthroscopic medial release, knee arthroplasty, painful prosthesis, medial plica, knee osteoarthritis

Posted Date: December 23rd, 2020

DOl: https://doi.org/10.21203/rs.3.rs-132353/v1

License: (우 (i) This work is licensed under a Creative Commons Attribution 4.0 International License. Read Full License

Version of Record: A version of this preprint was published on January 19th, 2021. See the published version at https://doi.org/10.1186/s13018-020-02191-7. 


\section{Abstract}

Introduction: Persistent postoperative pain (PPOP) has detracted from some otherwise successful knee arthroplasties. This study investigated medial abrasion syndrome (MAS) as a cause of PPOP after knee arthroplasty. The surgical techniques and outcomes of incorporating this concept into the management of both primary arthroplasty cases and patients suffering from unknown causes of PPOP after arthroplasties were presented.

Materials and Methods: In a one-year period, the author performed unicompartmental or total knee arthroplasty (the UKA or TKA group) that also eliminated medial abrasion phenomenon (MAP) on 196 knees of 150 patients at advanced stages of knee osteoarthritis (OA). During the same year, 16 knees of 16 patients with unknown causes of PPOP after knee arthroplasties were referred to the author for the arthroscopic medial release procedure (the AMR group) after being diagnosed as MAS. Subjective satisfaction, Knee Society Score (KSS), and Knee injury and Osteoarthritis Outcome Score (KOOS) evaluations were used for outcome study.

Results: All 166 patients were followed for more than 3 years (mean: 3.7 years, 3.1-4.2) for outcome study. All knees receiving arthroplasty showed medial plicae with MAP at the time of surgery. Only 2 of them suffered from PPOP: one was a neglected tibial plateau fracture with residual varus deformity after UKA and the other was a late infection after TKA and received revision. The satisfactory rate was $98.8 \%$ in the UKA group, $99.1 \%$ in the TKA group, and $100 \%$ in the AMR group. The Knee Society scores and all subscales of KOOS were statistically improved in all groups.

Conclusions: MAS is a cause of pain in patients who have received knee arthroplasties, and MAP should be eliminated to ensure a successful knee arthroplasty. PPOP after knee arthroplasty can be caused by MAS, which can be managed by AMR.

\section{Background}

Knee arthroplasty has generally been highly successful when judged by prosthesis-related outcomes. However, persistent postoperative pain (PPOP) has been a common occurrence in about $10-53 \%$ of patients after a total knee arthroplasty (TKA) and is associated with reduced health-related quality of life [1]-[5]. Studies regarding unicompartmental knee arthroplasty (UKA) also reported a high incidence of medial knee discomfort and pain which did not correlate with the postoperative radiographic scores, preoperative arthritis, and the positioning of the prosthesis and might lead to unnecessary revisions [6]-[9].

Many factors may be responsible for a painful knee prosthesis. Common causes of prosthetic failure, such as aseptic loosening, infection, instability, progressive patellar arthropathy and recurrent synovitis are associated with clearly defined radiographic and/or clinical evidence [10], [11]. Nevertheless, it can be extremely difficult to diagnose and treat a painful knee prosthesis if there is no clear evidence of any of those most common causes of failure. In cases of unexplained pain, reoperation is unwise and frequently associated with suboptimal results [12], [13]. 
Since medial abrasion phenomenon (MAP) related medial abrasion syndrome (MAS) is a common cause of knee pain in middle and old age with knee OA [14], its role as a cause of PPOP after a knee arthroplasty cannot be disregarded. We have therefore included the elimination of the MAP in our knee arthroplasty procedures since 2010. In this study, we retrospectively evaluated our case series in a one-year period for the incidence of PPOP after knee arthroplasties incorporating the technique of MAP elimination. The outcomes of arthroscopic medial release (AMR) [15] for referred patients with unknown causes of PPOP during the same time period was also investigated. We postulate that MAS is a cause of PPOP after knee arthroplasty and it could be treated with AMR.

\section{Materials And Method}

In a one-year duration (2015), 196 knees of 150 patients at advanced stages of knee OA received arthroplasty (UKA: 80 knees of 66 patients, TKA: 116 knees of 84 patients) by the first author. In the same year, 16 knees of 16 patients with unknown causes of PPOP after knee arthroplasty (UKA: 2 knees, TKA: 14 knees) were referred to our service from other hospitals for the AMR management. These patients were prospectively followed as part of an Institutional Review Board Registry. The distribution of age, sex, the main compartment involved, and the type of arthroplasty performed on these knees are listed in Table 1. For the arthroplasty group, the inclusion criteria were advanced primary OA (stage IV or V according to Lyu's clinical classification [16]). UKA was performed for stage IV or V OA involving only one compartment. For stage IV or V OA involving more than one compartment, TKA was undertaken. All patients who received AMR were referred to our service from other institutions with the diagnosis of PPOP of unknown causes after their knee arthroplasty and had been treated conservatively, including physical therapy and medication, for more than one year. Before AMR was performed, evidence of any clearly defined common causes of prosthetic failure including aseptic loosening, instability, progressive patellar arthropathy, infection and recurrent synovitis were ruled out by radiographic and laboratory examinations. Typical symptoms and signs of pain, crepitus, snapping, localized tenderness or palpable band described in previous report [14] confirmed the diagnosis of MAS before surgery.

Table 1

Age, sex and main involved compartment distribution in different type of surgery

\begin{tabular}{|c|c|c|c|c|c|c|c|c|}
\hline UKA & & & TKA & & & AMR & & \\
\hline $\begin{array}{l}\text { Age (SD) } \\
\text { No. }\end{array}$ & $\begin{array}{l}\text { F/M } \\
\text { (Ratio) }\end{array}$ & $\begin{array}{l}\text { Med./Lat. } \\
\text { (Ratio) }\end{array}$ & $\begin{array}{l}\text { Age (SD) } \\
\text { No. }\end{array}$ & $\begin{array}{l}\text { F/M } \\
\text { (Ratio) }\end{array}$ & $\begin{array}{l}\text { Med./Lat. } \\
\text { (Ratio) }\end{array}$ & $\begin{array}{l}\text { Age } \\
\text { (SD) } \\
\text { No. }\end{array}$ & $\begin{array}{l}\text { F/M } \\
\text { (Ratio) }\end{array}$ & $\begin{array}{l}\text { TKA/UKA } \\
\text { (Ratio) }\end{array}$ \\
\hline $\begin{array}{l}72.1 \\
(7.8) \\
80\end{array}$ & $\begin{array}{l}53 / 27 \\
(2.0)\end{array}$ & $\begin{array}{l}80 / 0 \\
-\end{array}$ & $\begin{array}{l}74.0 \\
(6.6) \\
116\end{array}$ & $\begin{array}{l}95 / 21 \\
(4.5)\end{array}$ & $\begin{array}{l}104 / 12 \\
(8.7)\end{array}$ & $\begin{array}{l}76.3 \\
(5.7) \\
16\end{array}$ & $\begin{array}{l}15 / 1 \\
(15)\end{array}$ & $\begin{array}{l}14 / 2 \\
(7)\end{array}$ \\
\hline
\end{tabular}




\section{Surgical procedures for arthroplasty}

For UKA, all cases were performed with the same cemented, metal backed fixed bearing implant (ZUK; Zimmer, Warsaw, IN, USA); for TKA, cemented posterior-stabilized implant (NexGen LPS-flex fixed knee system; Zimmer, Warsaw, IN, USA) was used in all cases. All arthroplasties were performed via a straight anterior incision with a medial parapatellar approach. For UKA, a tibia-first extension gap balancing technique was used. For TKA, the technique utilizing intramedullary femoral and extramedullary tibial alignment guides was followed. After the installation of prosthesis, the elimination of existing MAP was performed before wound closure. Various severity of pathologic medial plica described in previous literature [17] could be identified in the medial gutter. As shown in Fig. 1 and Additional file 1: Video 1, the thickened medial plica was removed completely from the attachment of genu articularis to the tendon sheath of pes anserinus.

\section{Surgical procedure for arthroscopic medial release}

During arthroscopic examination, remnants or fibrosis of medial plica could be identified over the inferiormedial aspect of the patellofemoral joint (PFJ) as shown in Fig. 2a. Tightness of the PFJ and impingement of the fibrotic medial plica were also verified (Fig. 2b). AMR was then performed as shown in Additional file 2: Video 2. The adequacy of the medial release was checked by pushing the tip of the scope under the patella and verifying if the previously tightly closed medial PFJ space could be easily opened and the medial retinaculum was clearly visible when the knee was fully extended (Fig. 2c).

\section{Post-operative Management}

Suction drain was used for all patients. Below-knee stockings to prevent thromboembolic disease for both lower limbs were used. Full range of motion and free ambulation were allowed as tolerated. After discharge from the hospital, home exercise programs, including active range of motion (knee hug and knee press) and quadriceps setting, were emphasised.

\section{Follow-up and Evaluation of Outcomes}

Regular follow-ups were undertaken monthly for 6 months. Thereafter, patients returned yearly for outcome evaluation including clinical and radiographic examinations. The comparisons of both pre- and postoperative Knee Society score (KSS) and knee injury and osteoarthritis outcome score (KOOS) were used for outcome evaluation. Subjective satisfaction was assessed by direct question using a categorical scale prepared for this study: excellent, free of symptoms, no limitation in activities; good, greatly improved, occasional pain, normal activities; fair, same as pre-operative condition, no improvement; and poor, has received or considered further operative treatment. The outcome was regarded as satisfactory if subjective satisfaction was rated as "excellent" or "good". The inquiry into subjective satisfaction and the evaluation of KSS and KOOS were conducted by nursing specialists. All investigations focused on individual knees in bilateral cases.

\section{Statistical Evaluation}


All values were presented with means and standard deviations. Statistical analysis for comparing preoperative and postoperative KSS and KOOS was performed using the paired $t$ test. $P<0.05$ was considered to be statistically significant. All statistical analysis was carried out using JMP, the Statistical Discovery Software (Version 5.0.1.2, SAS Institute Inc., Cary, NC, USA).

\section{Results}

All patients were successfully followed for more than 3 years (mean: 3.7 years, 3.1-4.2) and were available for outcome study including subjective satisfaction, KSS, and KOOS evaluations. Medial plicae with MAP were identified in all the knee that received arthroplasty. Two patients suffered from PPOP: one patient in the UKA group had persistent pain due to a neglected tibial plateau fracture with residual varus deformity, the only dissatisfied patient in the TKA group suffered from a late infection with persistent pain and received revision. The satisfactory rate was $98.8 \%$ in the UKA group, $99.1 \%$ in the TKA group, and $100 \%$ in the AMR group (Table 2). The Knee Society scores and all subscales of KOOS were statistically improved in all groups (Tables 3 and 4). No evidence of loosening or wearing of the prostheses was found by radiographic examinations in all groups.

Table 2

Subjective outcomes stratified by type of surgery

\begin{tabular}{|llllll|}
\hline Operation (N) & E & G & F & P & Sat. (\%) \\
\hline AMR (16) & $10(62.5)$ & $6(37.5)$ & 0 & 0 & 100.0 \\
UKA (80) & $74(92.5)$ & $5(6.3)$ & 0 & $1(1.2)$ & 98.8 \\
\hline TKA (116) & 108 (93.1) & $7(6.0)$ & 0 & $1(0.9)$ & 99.1 \\
\hline † E: excellent; G: good; F: fair; P: poor; Sat.: satisfied = E + G \\
\hline
\end{tabular}

Table 3

Pre-operative and post-operative Knee Society score for different operation

\begin{tabular}{|lllll|}
\hline Operation (N) & Pain (SD) & \multicolumn{3}{l|}{ Function (SD) } \\
\cline { 2 - 5 } & Pre-op. & Post-op. & Pre-op. & Post-op. \\
\hline AMR (16) & $55.4(11.5)$ & $90.6(9.3)$ & $47.8(15.8)$ & $79.4(14.7)$ \\
\hline Uni-K (80) & $60.7(13.6)$ & $92.7(9.0)$ & $46.2(17.3)$ & $82.9(15.2)$ \\
\hline TKA (116) & $53.1(19.6)$ & $95.8(5.8)$ & $38.2(20.0)$ & $80.2(16.8)$ \\
\hline P value & $<0.001$ & & $<0.001$ & \\
\hline
\end{tabular}


Table 4

Pre-operative and post-operative KOOS for different operation

\begin{tabular}{|c|c|c|c|c|c|c|c|c|c|c|}
\hline & $P$ & & $S$ & & $A D L$ & & $S / R$ & & QOL & \\
\hline $\begin{array}{l}\text { Operation } \\
(\mathrm{N})\end{array}$ & $\begin{array}{l}\text { Pre- } \\
\text { op. } \\
\text { (SD) }\end{array}$ & $\begin{array}{l}\text { Post- } \\
\text { op. } \\
\text { (SD) }\end{array}$ & $\begin{array}{l}\text { Pre- } \\
\text { op. } \\
\text { (SD) }\end{array}$ & $\begin{array}{l}\text { Post- } \\
\text { op. } \\
\text { (SD) }\end{array}$ & $\begin{array}{l}\text { Pre- } \\
\text { op. } \\
\text { (SD) }\end{array}$ & $\begin{array}{l}\text { Post- } \\
\text { op. } \\
\text { (SD) }\end{array}$ & $\begin{array}{l}\text { Pre- } \\
\text { op. } \\
\text { (SD) }\end{array}$ & $\begin{array}{l}\text { Post- } \\
\text { op. } \\
\text { (SD) }\end{array}$ & $\begin{array}{l}\text { Pre- } \\
\text { op. } \\
\text { (SD) }\end{array}$ & $\begin{array}{l}\text { Post- } \\
\text { op. } \\
\text { (SD) }\end{array}$ \\
\hline \multirow[t]{2}{*}{ AMR (16) } & 54.3 & 93.7 & 46.6 & 77.7 & 63.8 & 89.4 & 16.1 & 38.4 & 34.0 & 66.0 \\
\hline & $(14.4)$ & $(7.3)$ & $(15.9)$ & (15.2) & $(9.4)$ & $(10.5)$ & $(18.6)$ & $(31.2)$ & (19.2) & (24.7) \\
\hline \multirow{2}{*}{$\begin{array}{l}\text { Uni-K } \\
(80)\end{array}$} & 53.3 & 93.7 & 47.8 & 86.9 & 60.2 & 89.5 & 22.8 & 61.9 & 37.7 & 82.1 \\
\hline & (18.1) & $(10.4)$ & (15.2) & (15.4) & $(18.4)$ & $(16.7)$ & $(21.4)$ & (35.3) & $(18.4)$ & (21.4) \\
\hline \multirow{2}{*}{$\begin{array}{l}\text { TKA } \\
(116)\end{array}$} & 50.5 & 95.9 & 46.1 & 89.0 & 56.3 & 93.9 & 22.7 & 60.4 & 33.1 & 84.1 \\
\hline & (17.7) & $(7.2)$ & $(15.1)$ & (12.3) & (19.3) & (8.3) & $(21.2)$ & $(30.7)$ & $(16.7)$ & (20.4) \\
\hline$P$ value & $<0.001$ & & $<0.001$ & & $<0.001$ & & $<0.001$ & & $<0.001$ & \\
\hline
\end{tabular}

\section{Discussion}

PPOP during everyday activities or at rest is a major cause of patient dissatisfaction after knee arthroplasty. It affects the quality of life of a significant percentage of patients who have undergone TKA [1]-[5], [18] and is also a common postoperative complaint after UKA [6]-[9]. According to the findings of this study, the elimination of MAP during either TKA or UKA could avoid unknown causes of PPOP during more than 3 years follow-up. Moreover, patients with unknown causes of PPOP after arthroplasties have become satisfied after their MAP were treated with AMR.

Many theories have been proposed to explain the etiology and risk factors of PPOP after knee arthroplasty [19], [20]. But, despite medical advances, unknown causes of PPOP has remained a clinical problem, and it is not clear why these conditions occur. It can be described as retropatellar or peripatellar pain, which limits patients in their everyday activities. Patients might experience difficulty in standing up from a chair, walking up and down stairs and riding a bicycle. Sometimes, even trying to put their knees in extension position is painful and difficult. This troublesome situation remains a challenge for the surgeons and usually leads to two questions: "How can a 'perfectly' placed knee arthroplasty (TKA or UKA) still be painful?" and "What may have caused this pain?" [19]. Some patients might therefore be claimed to have a higher than normal depressive or anxiety state [20].

To increase overall patient satisfaction, it is important to identify the different anatomical structures that can cause this pain so as to prevent PPOP after knee arthroplasty. MAS has been reported to be a neglected cause of knee pain in middle and old age with knee $O A$, and it can be effectively treated with AMR [14]. Incorporation of the elimination of MAP in arthroplasty has significantly decreased the incidence 
of PPOP in this series compared to previous literatures [1]-[9], [18]. For referred cases with unknown causes of PPOP, we also found that AMR could deliver satisfactory outcomes for the patients. Both of these findings have shone light on the important role that MAP plays in causing pain after arthroplasty.

Arthroscopy for failed knee arthroplasty is a well-documented and accepted procedure for the diagnosis of component and soft tissue problems [10], [21]-[23]. It is a valuable tool to evaluate a painful knee arthroplasty, and it can be used to treat certain conditions such as removing loose bodies, correcting patella subluxation with a lateral release, excising a symptomatic pseudomeniscus, and releasing intraarticular adhesions to improve motion and relieve pain that would otherwise require an arthrotomy [24]. However, MAS and AMR have not been reported in the literature regarding PPOP. Our study has broadened the therapeutic value of arthroscopy for patients with PPOP.

There are some limitations in this study. First, it is a retrospective study of a consecutive case series and there was not a comparative group. Nevertheless, the significant low incidence of PPOP after arthroplasty incorporating elimination of MAP in this series compared to other reports [1]-[9] still provides evidence to our hypothesis that MAS is a cause of PPOP. Second, as this is a case series by a single surgeon who utilized the same surgical technique in every case, we are unable to assess other factors such as the approaches and the extent of fat pad resection which might associated with PPOP after arthroplasty. Third, the low number of patients who received AMR for PPOP with unknown causes may have overemphasized the incidence of MAS as a cause of PPOP. However, these drawbacks might draw attention for further investigations.

\section{Conclusion}

MAS is a neglected cause of knee pain in patients who have received arthroplasties. Elimination of MAP during knee arthroplasty could lower the incidence of PPOP. Unrecognized and untreated MAP in arthroplasty is a cause of PPOP and it can be managed by AMR.

\section{Abbreviations}

$\mathrm{PPOP}=$ persistent postoperative pain

MAS = medial abrasion syndrome

AMR $=$ arthroscopic medial release

UKA = unicompartmental knee arthroplasty

TKA = total knee arthroplasty

$\mathrm{OA}=$ osteoarthritis

KSS $=$ knee society score 
KOOS = knee injury and osteoarthritis outcome score

MAP $=$ medial abrasion phenomenon

PFJ = patellofemoral joint

\section{Declarations}

\section{Ethics approval and consent to participate}

All patients participated in this study were prospectively followed up as part of an Institutional Review Board Registry in Taiwan (IRB Approval Number: B09704022). Written informed consent was obtained from all the participants.

\section{Availability of data and materials}

The datasets during and/or analyzed during the current study are available from the corresponding author on reasonable request.

\section{Competing interests}

The authors declare that they have no competing interests.

\section{Funding}

No funding was received.

\section{Authors' contributions}

SRL was involved in the design of the study and performed the operations. $\mathrm{CCH}$ and JPH collected data and performed the statistical analysis. SRL and $\mathrm{CCH}$ were responsible for drafting the paper and revising it. All authors have read and approved the final manuscript.

\section{Acknowledgements}

None.

\section{References}

1. A. D. Beswick, V. Wylde, R. Gooberman-Hill, A. Blom, and P. Dieppe, "What proportion of patients report long-term pain after total hip or knee replacement for osteoarthritis? A systematic review of prospective studies in unselected patients.," BMJ Open, vol. 2, no. 1, p. e000435, Jan. 2012.

2. S. S. Liu et al., "A cross-sectional survey on prevalence and risk factors for persistent postsurgical pain 1 year after total hip and knee replacement.," Reg. Anesth. Pain Med., vol. 37, pp. 415-22, 2012. 
3. S. T. Skou, T. Graven-Nielsen, S. Rasmussen, O. H. Simonsen, M. B. Laursen, and L. Arendt-Nielsen, "Facilitation of pain sensitization in knee osteoarthritis and persistent post-operative pain: A crosssectional study," Eur. J. Pain (United Kingdom), 2014.

4. R. B. Bourne, B. M. Chesworth, A. M. Davis, N. N. Mahomed, and K. D. J. Charron, "Patient satisfaction after total knee arthroplasty: Who is satisfied and who is not?," in Clinical Orthopaedics and Related Research, 2010.

5. S. J. M. Breugem, B. van Ooij, D. Haverkamp, I. N. Sierevelt, and C. N. van Dijk, "No difference in anterior knee pain between a fixed and a mobile posterior stabilized total knee arthroplasty after 7.9 years," Knee Surgery, Sport. Traumatol. Arthrosc., 2014.

6. M. C. Edmondson, D. Isaac, M. Wijeratna, S. Brink, P. Gibb, and P. Skinner, "Oxford unicompartmental knee arthroplasty: Medial pain and functional outcome in the medium term," J. Orthop. Surg. Res., 2011.

7. P. N. Baker, T. Petheram, P. J. Avery, P. J. Gregg, and D. J. Deehan, “Revision for unexplained pain following unicompartmental and total knee replacement," J. Bone Jt. Surg. - Ser. A, 2012.

8. D. A. Crawford, K. R. Berend, and A. V. Lombardi, "Management of the Failed Medial Unicompartmental Knee Arthroplasty," The Journal of the American Academy of Orthopaedic Surgeons. 2018.

9. J. A. Kennedy et al., "Most unicompartmental knee replacement revisions could be avoided: a radiographic evaluation of revised Oxford knees in the National Joint Registry," Knee Surgery, Sport. Traumatol. Arthrosc., 2020.

10. H.-M. Klinger, M. H. Baums, G. Spahn, and T. Ernstberger, "A study of effectiveness of knee arthroscopy after knee arthroplasty.," Arthroscopy, vol. 21, no. 6, pp. 731-8, Jun. 2005.

11. M. Villano, C. Carulli, S. Puccini, S. Soderi, and M. Innocenti, “Painful knee prosthesis: surgical approach.," Clin. Cases Miner. Bone Metab., vol. 8, no. 2, pp. 26-8, May 2011.

12. D. A. Dennis, “Evaluation of painful total knee arthroplasty.,” J. Arthroplasty, vol. 19, no. 4 Suppl 1, pp. 35-40, Jun. 2004.

13. A. D. Toms, V. Mandalia, R. Haigh, and B. Hopwood, "The management of patients with painful total knee replacement.," J. Bone Joint Surg. Br., vol. 91, no. 2, pp. 143-50, Feb. 2009.

14. S.-R. Lyu, C.-C. Lee, and C.-C. Hsu, "Medial abrasion syndrome: A neglected cause of knee pain in middle and old age," Med. (United States), vol. 94, no. 16, 2015.

15. S.-R. Lyu, "Arthroscopic medial release for medial compartment osteoarthritis of the knee: the result of a single surgeon series with a minimum follow-up of four years.," J. Bone Joint Surg. Br., vol. 90, no. 9, pp. 1186-1192, Sep. 2008.

16. S.-R. Lyu, "Knee health promotion option for knee osteoarthritis: A preliminary report of a concept of multidisciplinary management," Heal. Aging Res., 2015.

17. S.-R. Lyu, J.-K. Chiang, and C.-E. Tseng, "Medial plica in patients with knee osteoarthritis: A histomorphological study," Knee Surgery, Sport. Traumatol. Arthrosc., vol. 18, no. 6, 2010.

18. I. Grosu, P. Lavand'homme, and E. Thienpont, “Pain after knee arthroplasty: An unresolved issue," Knee Surgery, Sports Traumatology, Arthroscopy. 2014. 
19. S. J. Martinus Breugem and D. Haverkamp, "Anterior knee pain after a total knee arthroplasty: What can cause this pain?," World Journal of Orthopaedics. 2014.

20. M. P. Bonnin, L. Basiglini, and H. A. P. Archbold, "What are the factors of residual pain after uncomplicated TKA?," Knee Surgery, Sport. Traumatol. Arthrosc., 2011.

21. J. R. Bocell, C. D. Thorpe, and H. S. Tullos, "Arthroscopic treatment of symptomatic total knee arthroplasty.," Clin. Orthop. Relat. Res., no. 271, pp. 125-34, Oct. 1991.

22. S. A. Wasilewski and U. Frankl, "Arthroscopy of the painful dysfunctional total knee replacement.," Arthroscopy, vol. 5, no. 4, pp. 294-7, Jan. 1989.

23. J. W. K. Wong, P. W. P. Yau, and P. K. Y. Chiu, "Arthroscopic treatment of patellar symptoms in posterior stabilized total knee replacement.," Int. Orthop., vol. 26, no. 4, pp. 250-2, Jan. 2002.

24. D. R. Johnson, R. J. Friedman, J. B. McGinty, J. L. Mason, and E. W. St Mary, "The role of arthroscopy in the problem total knee replacement.," Arthroscopy, vol. 6, no. 1, pp. 30-2, Jan. 1990.

\section{Figures}




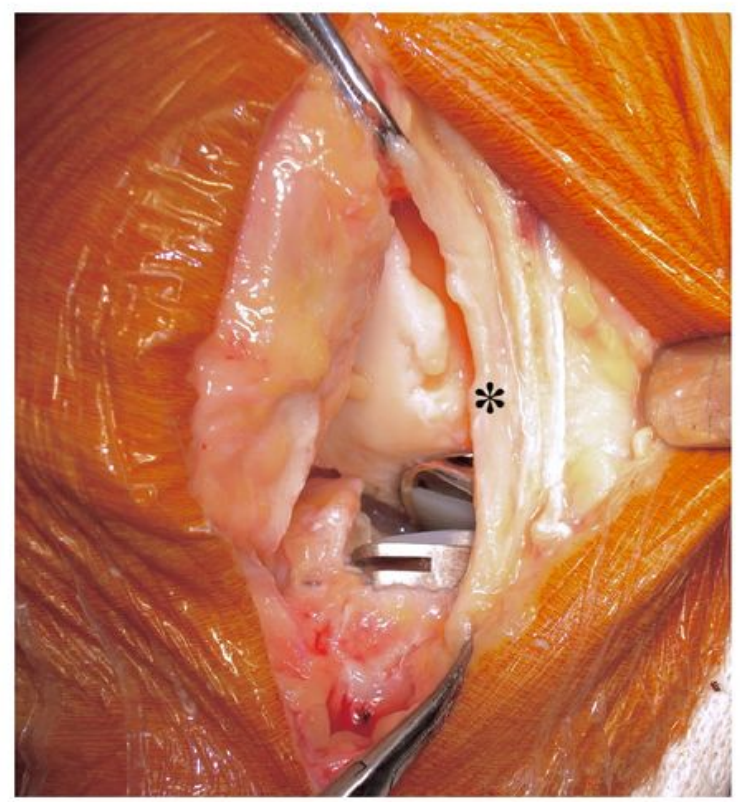

a

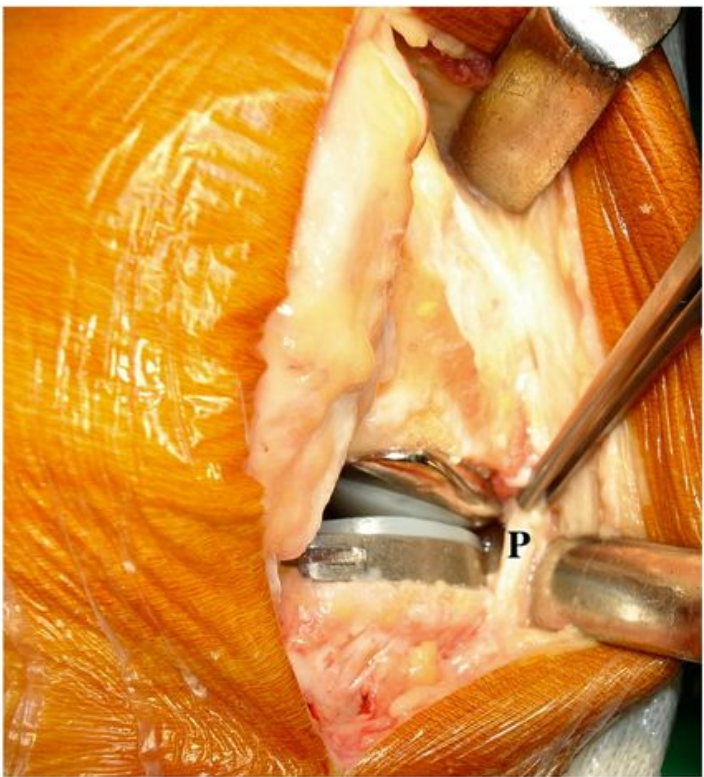

C

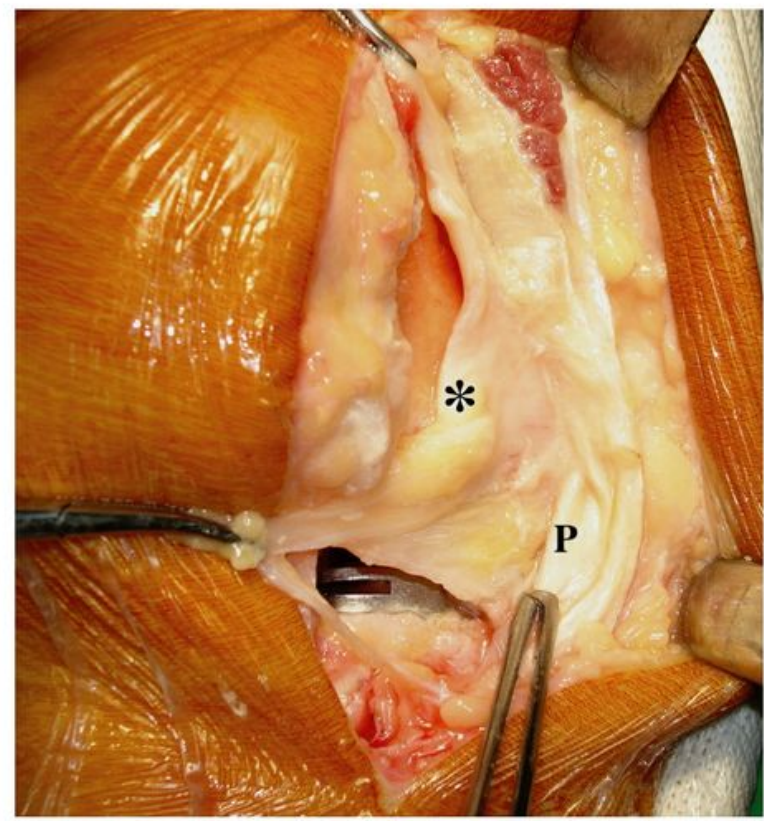

b

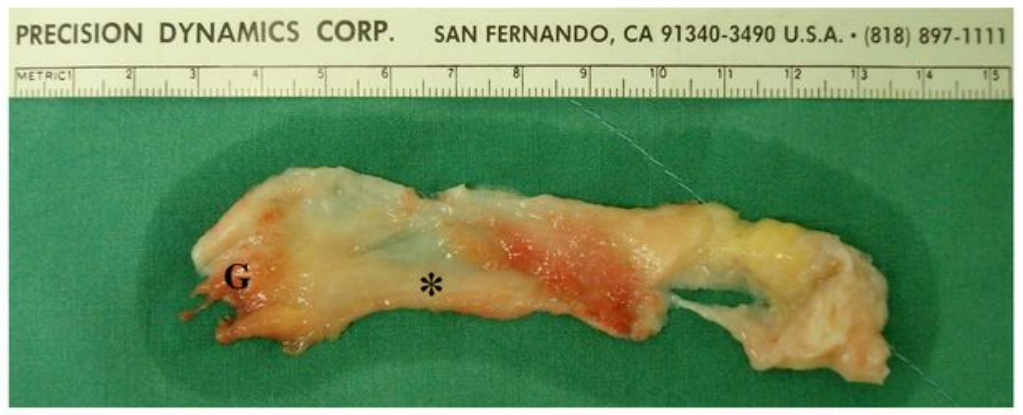

d

\section{Figure 1}

Elimination of medial abrasion phenomenon a, before wound closure, medial plica ${ }^{\star}$ ) was identified in the medial gutter; $b$, the synovial fold of the distal part of the medial plica $\left(^{\star}\right)$ was found to have originated from the tendon sheath of the pes anserinus $(P) ; C$, after the medial plica was completely removed, the prosthesis was clearly visible; $d$, various degrees of wear and fibrillation could be found along the margin of the medial plica, which was always hypertrophied and sometimes became cord-like $\left({ }^{\star}\right)$, a small branch of skeletal muscle originating from genu articularis $(G)$ was found in all knees. 

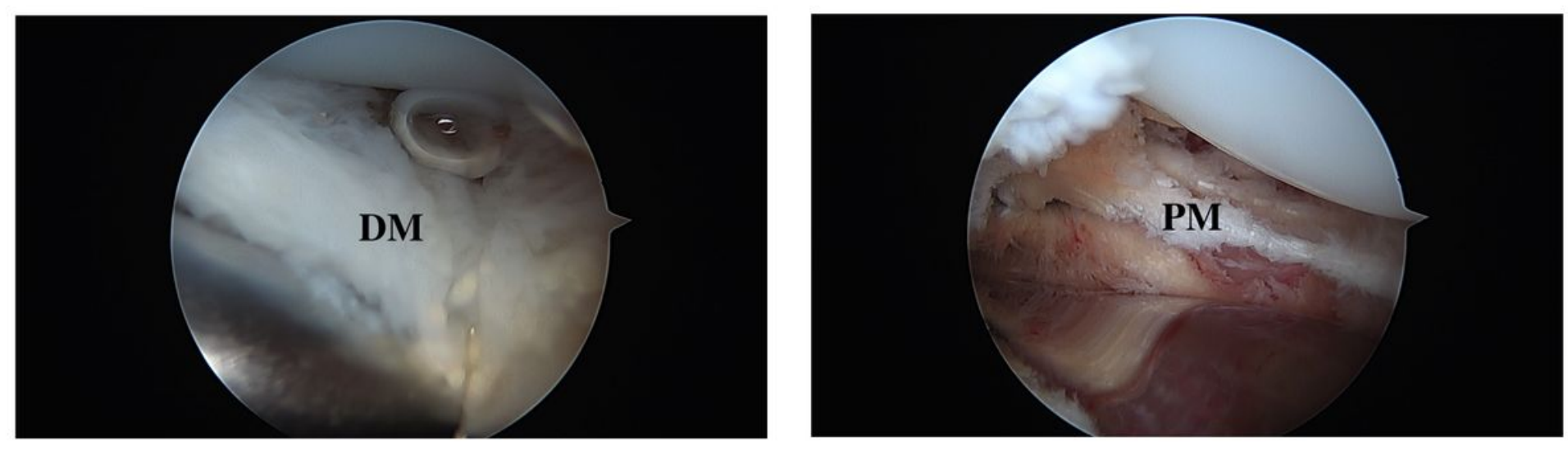

b

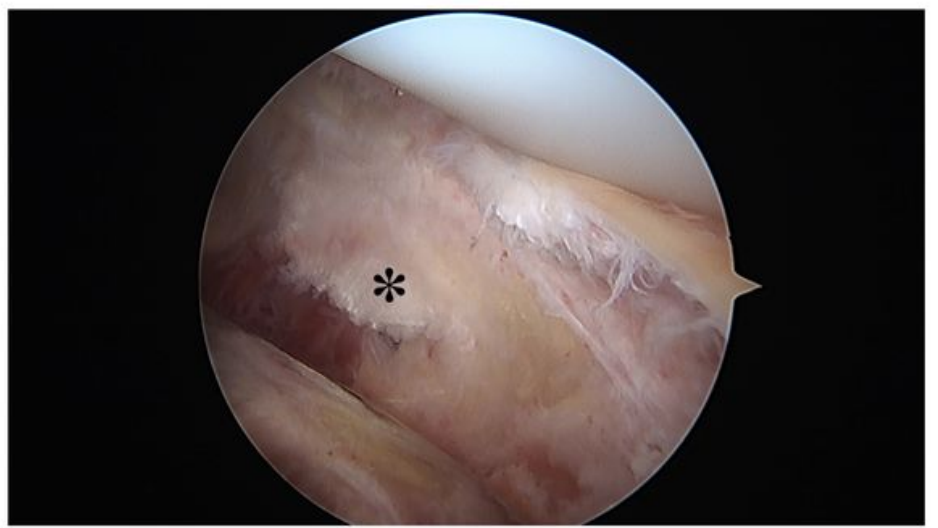

\section{Figure 2}

Arthroscopic findings when performing AMR in a patient with PPOP a \& b, before AMR, the patello-femoral joint was tight and fibrotic medial plica was found in the medial gutter (DM: distal medial plica, PM: proximal medial plica); c, after AMR, the tension of the patello-femoral joint was released and the medial retinaculum $\left(^{*}\right)$ was clearly visible.

\section{Supplementary Files}

This is a list of supplementary files associated with this preprint. Click to download.

- Video1EliminationofMAP.mp4

- Video2AMRforMASinpatientwithPPOP.mp4 